\title{
Correction to: Multi-scale imaging as an essential tool for precision medicine
}

\author{
Michael Phelps ${ }^{1}$ - Markus Schwaiger ${ }^{2}$ - Arturo Chiti ${ }^{3,4}$
}

Published online: 11 May 2021

(C) Springer-Verlag GmbH Germany, part of Springer Nature 2021

\section{Correction to: Eur J Nucl Med Mol Imaging https://doi.org/10.1007/s00259-021-05367-3}

The authors regret that they need to make some changes in the body of the original editorial article to make it clearer and readable to the readers.

The original article has been corrected.

The original article can be found at https://doi.org/10.1007/ s00259-021-05367-3.

Publisher's note Springer Nature remains neutral with regard to jurisdictional claims in published maps and institutional affiliations.

This article is part of the Topical Collection on Erratum.

The online version of the original article can be found at https://doi.org/ $10.1007 / \mathrm{s} 00259-021-05367-3$

Arturo Chiti

arturo.chiti@hunimed.eu

1 UCLA GPB, 23-138 CHS, 10833 Le Conte Ave., Los Angeles, CA 90095, USA

2 Klinikum rechts der Isar, Technical University of Munich, Ismaninger Straße 22, 81675 Munich, Germany

3 Department of Biomedical Sciences, Humanitas University, Via Rita Levi Montalcini 4, 20090 Pieve Emanuele, Milan, Italy

4 IRCCS Humanitas Research Hospital, Via Manzoni 56, 20089 Rozzano, Milan, Italy 